\title{
Increasing the Safety of People Activity in Aggressive Potential Locations, Analyzed through the Probability Theory, Modeling/Simulation and Application in Underground Coal Mining
}

\author{
Emil Pop, Gabriel-Ioan Ilcea, Ionut-Alin Popa, Lorand Bogdanffy \\ Department of Control Engineering, Computers, Electrical Engineering and Power Engineering, University of Petroșani, \\ Petroșani, Romania \\ Email:emilpop@upet.ro,gabi_ilcea@yahoo.com,ionutalin_popa@yahoo.ro, lorandbogdanffy@upet.ro
}

How to cite this paper: Pop, E., Ilcea, G.-I., Popa, I.-A. and Bogdanffy, L. (2019) Increasing the Safety of People Activity in Aggressive Potential Locations, Analyzed through the Probability Theory, Modeling/Simulation and Application in Underground Coal Mining. Engineering, 11, 93106.

https://doi.org/10.4236/eng.2019.112008

Received: December 19, 2018

Accepted: February 10, 2019

Published: February 13, 2019

Copyright $\odot 2019$ by author(s) and Scientific Research Publishing Inc. This work is licensed under the Creative Commons Attribution International License (CC BY 4.0).

http://creativecommons.org/licenses/by/4.0/

\begin{abstract}
This paper deals with the increasing safety of working in aggressive potential locations, having SCADA system and WSN sensors, using a "probabilistic strategy" in comparison with a "deterministic" one, modeling/simulation and application in underground coal mining. In general, three conditions can be considered: 1) an unfriendly environment that facilitates the risk of accidents, 2) aggressive equipments that can compete to cause accidents and 3) the work security breaches that can cause accidents. These conditions define the triangle of accidents and are customized for an underground coal mining where the methane gas is released with the exploitation of the massive coal. In this case, the first two conditions create an explosive potential atmosphere. To allow people to work in a safe location it needs: first, a continuing monitoring through SCADA system of the explosive potential atmosphere and second, the use of antiexplosive equipment. This method, named "deterministic strategy", increases the safety of working, but the explosions have not been completely eliminated. In order to increase the safety of working, the paper continues with the presentation of a new method based on hazard laws, named "probabilistic strategy". This strategy was validated through modeling/simulation using CupCarbon software platform, and application of WSN networks implemented on Arduino equipments. At the end of the paper the interesting conclusions are emphases which are applicable to both strategies.
\end{abstract}

\section{Keywords}

Accident Potentially, Safety Zone, Triangle of Accidents, Hazard Laws, 
Deterministic Strategy, Probabilistic Strategy, CupCarbon Modeling and Simulation, WSN Applications, Arduino Implementation Example

\section{Introduction}

\subsection{Triangle of Explosions and Safe Location}

As stated [1], in some activities, staff must work in an unfriendly or even aggressive zone where the environment in which the activity unfolds can affect the health and safety of the people. These are risks such as explosions, physical injuries, health and illness, accidents, etc. These situations exist in some sectors: mining, military, marine, oil and gas, construction etc. For example, in coal extractions, a mixture of air (oxygen) and methane produces an atmosphere that can explode in the present of a flame or spark. To characterize whether an area or location is potentially aggressive, three conditions need to be considered: 1) if there are phenomena or events that facilitate the occurrence of accidents; 2) if there are conditions permitting the unfolding of the events; 3 ) if there are sources of initiation of accidents at that location. These conditions define the triangle of accidents. We will define the following locations:

- Accident risk location is where all three conditions are present.

- The location with potential for accidents is where the first two conditions exist.

- A safe location is one where conditions (1) and (3) can never occur.

A location with potential for accidents can be maintained as a safe location if by technical means the conditions (1) and (3) are excluded. Work is only allowed in secure locations. Depending on the concrete case, the accident triangle can be customized as it will appear in the context for the case encountered in underground carbon mining [2].

The three conditions above are thus customized defining the so-called fire triangle:

- If there are certain highly flammable substances in the atmosphere, such as methane.

- If there is an atmosphere with enough oxygen to keep the flame and the explosion.

- If there are some sources of energy that can initiate the explosion, spark or flame.

Based on the above conditions for underground coal mining we have:

- Potentially explosive atmospheres in the case of the first two conditions.

- Explosive or hazardous atmosphere if all three conditions are present.

- A safe area where explosion cannot occur and cannot be transmitted outdoors to initiate explosion in potentially explosive atmospheres.

The presence of some substances (butane, propane, acetylene, methane, carbon monoxide, petroleum fuels, oils, chemical solvents, sugar, flour, cereals, 
plastics etc.) turns a normal location into one with a potentially explosive atmosphere [3].

Supplementary, the presence of ignition energy sources (hot surfaces, flame and hot gases, mechanical and/or electric sparks, electrical equipment, static electricity, electromagnetic waves, exothermic reactions, etc.) transforms a potentially explosive atmosphere, into an explosion-hazardous location. Electrical equipment is a source of ignition energy and therefore, to prevent the initiation of explosions, is specially manufactured in an antiexplosive construction.

An important category of antiexplosive electrical equipment, safe for any source of ignition (explosion), is intrinsically safe, and has positive safety, generating a stop signal for remote control port failures [2].

- Intrinsic safety provides a level of safety for electrical equipment that, in abnormal or normal operation, cannot initiate potentially explosive atmospheres.

- Antiexplosive/antideflagration is a safety level that an electrical equipment is included in a special case so that in normal or abnormal condition it cannot transmit from the inside out, enough energy to initiate a potentially explosive atmosphere.

- Hazardous area is an area where there is a risk of explosion.

- Safety barrier is a module that ensures that input/output is intrinsically safe.

- Positive input port security is a port quality of a remote-control system that, for any malfunction, generates a stop signal for the process.

The safety barrier isolates the dangerous area from the safe area. If a safe area is created using a special enclosure, the entrance and exit are connected by a safety barrier.

Any personal activity is allowed only in secure areas (locations). For example, in the case of coal mining underground, jobs are taking place in potentially explosive locations because methane is releasing once the coal is extracted from the coal massive. Transforming the potentially explosive location into a safe location is done through two actions as follows:

- Maintaining the percentage of methane in the atmospheres below a level that is non-hazardous.

- Eliminating any suspected explosion initiation from that location.

The first condition is ensured by airing the locations using ventilation and constant monitoring of the methane level and the second condition by using explosion-proof construction equipment. To preserve a safe location it is necessary to maintain the integrity of both conditions, which means using an automated methane monitoring and control system, and anti-explosive equipment that is regularly checked to maintain his integrity, by specialized personnel. This method has proven to be effective, accidents due to explosion risks have fallen sharply. In Figure 1 are illustrated the accident triangle and an antiexplosive electrical equipment, with intrinsic safety, which isolate the safe area from the hazardous area, using safety barrier. 


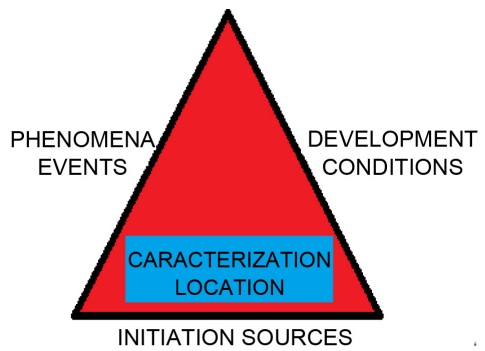

(a)

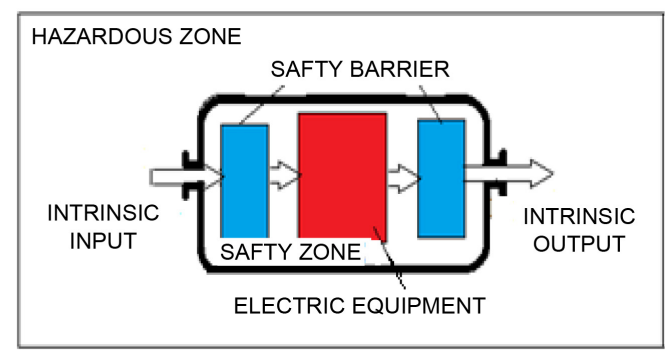

(b)

Figure 1. (a) Accident triangle; (b) Electrical explosion-proof barrier equipment.

\subsection{Increasing the Safety of People by Deterministic Strategy}

Mining for the extraction of coal and other mineral resources is considered an activity that due to the specificity of the works is very dangerous because of the occurrence of accidents with drastic consequences on human life and with great economic damage. Depending on the geological-mining conditions, extraction of coal from the massive is done through various exploitation methods. Of these, combine (2), mechanized complex (5) and conveyor (3) have the advantage of large productivity with reduced physical effort, as shown in Figure 2(a) [4].

As a result of the coal exploitation, empty spaces are created, called "exploited spaces" (5), where gases such as methane $\mathrm{CH}_{4}$ carbon monoxide $\mathrm{CO}$ and carbon dioxide $\mathrm{CO}_{2}$ accumulates. If the last two gases are met rarely, more in the case of mine fires, the methane is almost always present by accompanying the extraction of coal from the massive.

The presence of methane in mine working is dangerous first of all because it forms explosive mixtures with the air. The maximum intensity explosion occurs at a methane-air content of $9.5 \%$. If the methane concentration in the air is greater than $9.5 \%$ the combustion is incomplete resulting in carbon monoxide [5].

Coal extraction in respective of the method requires from the outset to organize the location as a "safe area". For this reason, gas releases that could create an explosive atmosphere must be controlled and maintained in a perceived percentage as non-hazardous, and equipment, plant and machinery must be explosion-proof. Under these conditions the area becomes a safe job, the activities can be carried out under normal conditions, even if the location was potentially explosive.

Based of presentation below was developed a method, named "deterministic strategy" to conserve the Safe location through eliminating the Risk of explosion. The risk of explosion can occur only in the following coincidence: there is methane in a hazardous percentage and there is a source of ignition. Methane sources from the mine are continuously coming from the coal massive that is being exploited but also from already exploited spaces. Severe methane removal measures are taken by continuous ventilation, maintaining a non hazardous inferior boundary. This limit is monitored by continuous scanning using a centralized SCADA system [6]. Equally, the equipment is periodically inspected to 


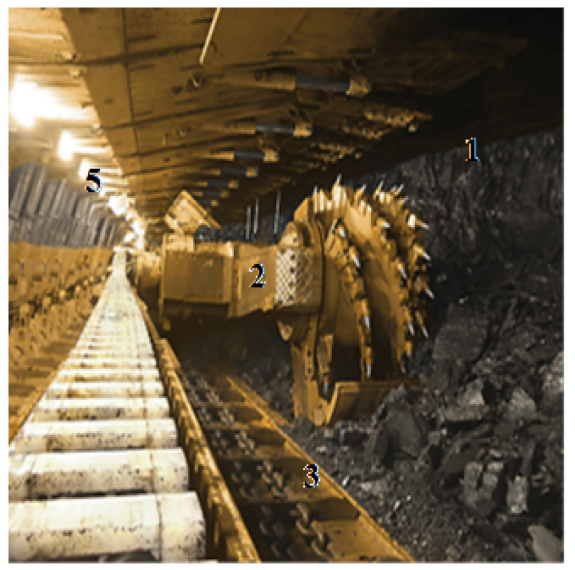

(a)

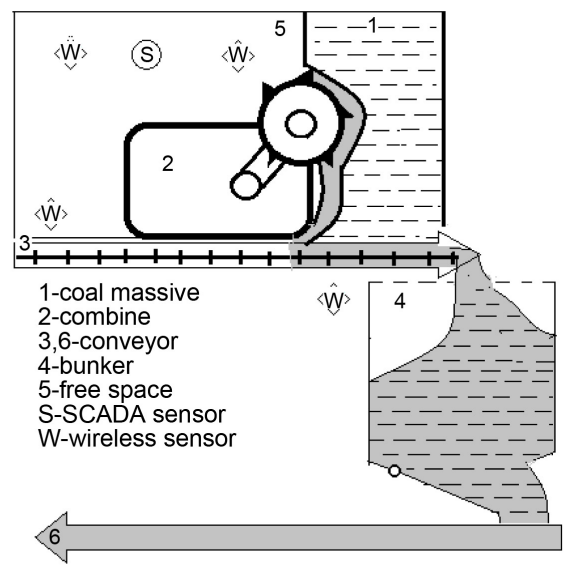

(b)

Figure 2. Exploitation of coal with complex, combines and conveyor (a); Simulation model (b).

have unaltered safety standards, (no sources of ignition).

Investigations into underground mining and geological research have concluded that systematic tracking in real time as well as maintaining machine and plant safety is a good way to prevent accidents and disasters. These systems have greatly reduced the explosion hazards but have not completely removed them so that unfortunately such incidents are still occurring. An unfortunate example is presented in the following example [7].

So many years ago, in the evening of November, a catastrophic explosion occurred at Livezeni mine in Jiu Valley, Romania. An entire production sector was affected, many miners were killed, others injured or blocked by the wave of rocks. The rescuers have worked continually for days and nights to free their comrades, but have not found survivors. The explosion resulted in 53 deaths, 27 injured and huge economic damages. The causes were difficult to determine and explain because the sector was assisted by dozens of gas detectors scanned continuously from the surface by SCADA [8]. The location was safe from the point of view of the risk of explosions. Although it seems totally unlikely to have the explosive atmosphere and spark of initiation at the same time, the catastrophe has occurred and since then, from time to time, the explosions have been both in the area and in other locations around the world. The question is why? In the next paragraph we try to answer to this question. The complexity of underground processes, the density of equipment's, the narrow workspace, the natural phenomena that are hard to avoid, the imperfections of some machines, the non-collaborative working of the SCADA scanning system, which allow the presence of security breaches between detectors, the non-use of human experience, some human errors, form the "random phenomena", where rare events, can occur. The random phenomena cannot be analyzed through deterministic methods but through the "hazard laws". They are the limits of "deterministic strategy".

A new method is proposed in this paper based on Hazard laws named "prob- 
abilistic strategy" which is developed in the next paragraph. This is based on "Gauss' bell" and "The law of large numbers". This approach permits to get an interesting result which will be validated by an application. This application use the network with three sensors, which sensing the uncovered hazardous space by the SCADA detectors, from exploited space and from cutting coal massive. The model of process is simulated by CupCarbon platform and based on results is implemented using Arduino equipments.

\section{Probabilistic Events Field and Hazard Laws}

\subsection{Probabilistic Event Field}

In the surrounding world, deterministic phenomena are quite few. Especially, most hazardous phenomena are random. Their study cannot be done by deterministic methods, and another method, known as the science of hazard, is needed. It has as a central element the frequency of the occurrence of a certain result (the ratio of the number of experiments in which the result appears and the number of all the experiments performed). If many experiments are performed, the frequency of occurrence of the result is its probability. The application of mathematics to the study of random phenomena is based on the fact that, by repeatedly repeating an experiment in virtually identical conditions, the frequency of occurrence oscillates around a constant number, called "average". The average is denoted by $\mathrm{m}$, and the oscillation distance (scattering) around the mean is called "average square deviation" and is denoted by $\sigma$. The degree of scattering around the mean is called "dispersion" and has the value of $\sigma^{2}$. In this way, an event $\mathrm{x}_{\mathrm{i}}$ can associate a number, namely the probability of producing it. In practice, we encounter many situations where a group of events happens randomly. Without giving a rigorous definition, we say that we are dealing with a "events field". The problem of converting an event field to numbers returns through define a numerical function that is a measure of the possibilities for realizing the events. We can assign a probability of occurring to each events and a probability function for the entire field. Thus, a "probabilistic events field" is obtained, to which the laws of the hazard apply. In a probabilistic events field, we can identify a random variable $X$ that produces events $X_{i}$, each with probability $p_{i}$. The function $p_{i}=f\left(X_{i}\right)$ is called the distribution law or the distribution of the random variable $X$ [9]. The best known is the Gauss-Laplace distribution law, known as Gauss' Bell because of his form, as a continuous function.

\subsection{Gauss' Bell}

This hazard law has the distribution function depending of " $m$ " and " $\sigma$ " and the expression:

$$
f(x)=\frac{1}{\sigma \sqrt{2 \pi}} \mathrm{e}^{\frac{-(x-m)^{2}}{2 \sigma^{2}}}
$$

In relation (1) $x$ is the event and $f(x)$ its probability. 
From Figure 3 it can be seen that the random variables are grouped around the average, for example at $m \pm \sigma$ the probability of occurrence is $68 \%$, and at a distance of $\pm 3.29 \sigma$ the probability falls below $1 / 1000$. In this case the event is very rare, it would seem impossible. Still under certain conditions these events happen and unfortunately with very serious consequences.

\subsection{The Law of Large Numbers}

Because we have little information, we can hardly determine the arithmetic mean of a number of random values. In fact, in the probabilistic event fields, the arithmetic mean of a sufficiently large number of events loses its character by chance and becomes secure. Probability' Law of Bernoulli, known as the "Law of Large Numbers", states that in a field of probabilistic events the arithmetic average of events is a constant " $\mathrm{m} / \mathrm{n}$ " if the number of attempts $\mathrm{n}$ is large enough.

The probability $P$, as the modulus of the difference between the relative frequency of the event $E$ in the case of the $n$ experiment ( $n$ sufficient large) from which we succeed $m$ and the probability $p$ of the event $E$, is lower than of the number $\mathcal{\varepsilon}$, positive arbitrarily.

$$
P(|m / n-p|<\varepsilon) \geq 1-1 / \varepsilon^{2} n
$$

Law of large numbers (theoretically): In the case of a large volume event field, if a $n$ volume selection is made and favourable results are obtained, then with a

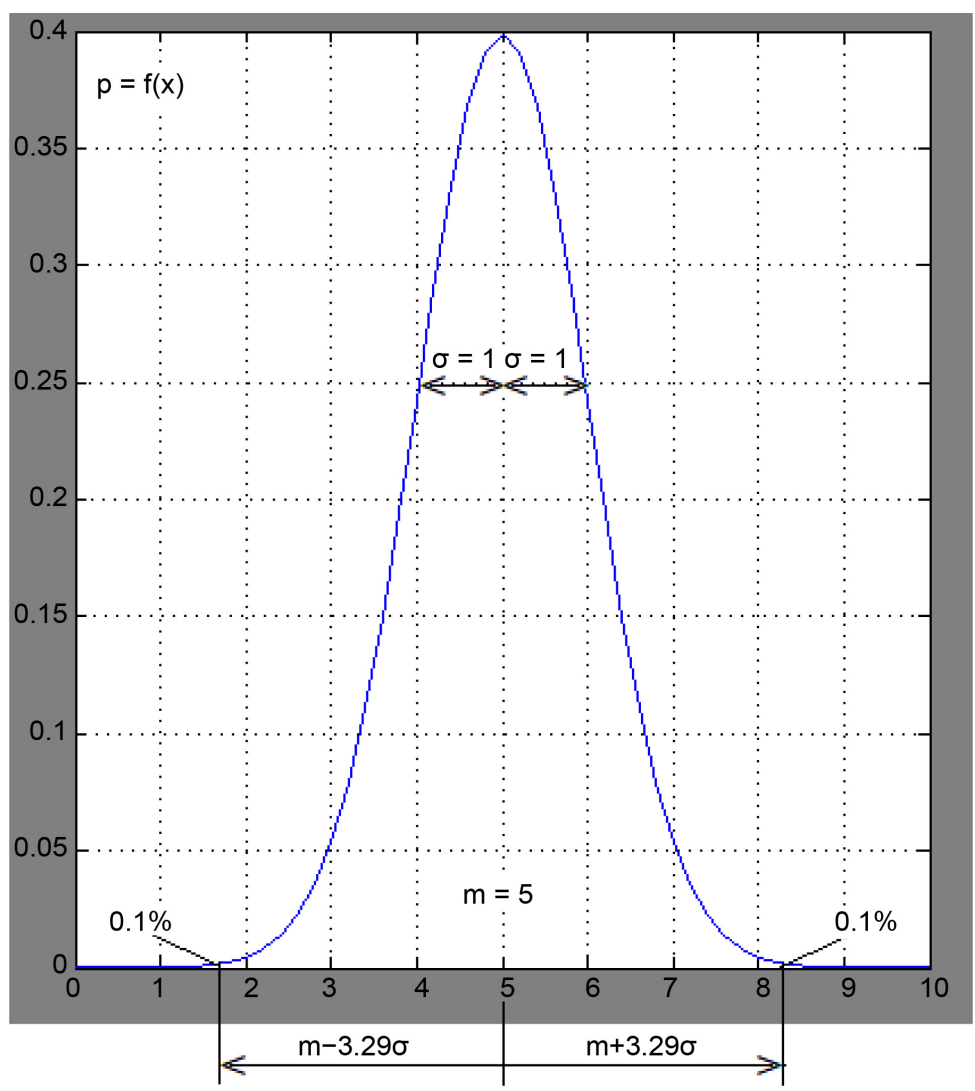

Figure 3. Gauss bell and random variable distribution mode $x$. 
probability close to the unit, we can say that the probability of the event is given by the relative frequency $\mathrm{m} / \mathrm{n}$. Therefore, if in the study of the phenomena for which we cannot determine a priori the probability $p$, it can be expressed experimentally by the relative frequency $m / n$ of the considered event, which is the theoretical justification of the use of the relative frequency instead of the probability. For practice it is important to know the conditions in which the combined action of several random factors leads to a result that is not random, so that we can predict the unfolding of the studied phenomenon. Such conditions are given in the theorems known as the law of large numbers.

Law of Large Numbers (Practical): Under the law of large numbers we will understand the assertion that, with a probability close to 1 , a certain event will occur, which depends on an arbitrarily large number of elementary events, each of which has a small influence on the phenomenon considered in the ensemble. For example, the experiment of throwing a coin of $n$ times and recording the number of occurrences of same face $k$, leads to $k / n=1 / 2$ if $\mathrm{n}$ is large enough. Mathematician Pearson threw a coin of $n=12,000$ times and obtained the value of $k=6019$ times, which gives a $k / n=0.5016$ frequency close to the probability of 0.5. A more practical version of the Law of Large Numbers is the following: If it is carried out in independent experiments, in each experiment the probability of $E$ is $p$, with the number of occurrences of event $E$ is $m$ and $\varepsilon \geq 0$ being an arbitrarily small number then:

$$
\lim _{n \rightarrow \infty} P\left(\left|\frac{m}{n}-p\right|<\varepsilon\right)=1
$$

In other words, if an experiment is repeated by a sufficiently large number of times $n$, under identical conditions and $m$ is the number of favorable results then the relative frequency is stable, that is $m / n$ and varies around the probability $p$.

\section{Developing the Probabilistic Strategies}

At the end of Paragraph 2, we presented a catastrophic explosion scenario at a coal mine where the location seemed safe in terms of accident risk, being assisted by dozens of gas detectors scanned continuously automatically from the surface by SCADA. Causes were difficult to determine because it seemed unlikely that there was explosive atmosphere and initiation spark at the same time. Although it seems totally unlikely to have the explosive atmosphere and spark of initiation at the same time, the catastrophe has occurred.

The complexity of underground processes, the density of equipment's, the narrow workspace, the natural phenomena that are hard to avoid, the imperfections of some machines, the non-collaborative working of the SCADA scanning system, which allow the presence of security breaches between detectors, the non-use of human experience, some human errors, form the "random phenomena", where rare events, can occur. The random phenomena cannot be analyzed through deterministic methods but through the "hazard laws". They are 
the limits of "deterministic strategy".

Let us say that in the "random phenomena" there is a "probabilistic events field" that takes place according to the hazard laws. In the following, we will present some consequences resulting from the hazard laws applicable in the case of catastrophic events like the above on which is based the Probabilistic strategies.

- It seems that by means of monitoring and controlling the safety of activities in potentially explosive areas is ensured up to the maximum limit of the Gauss' bell with the probability of $99.9 \%$. The uncovered Gaussian Bell Zone has the probability of $0.1 \%(1 / 1000)$ in which incident events are considered to be virtually impossible. The above statement is true if accident-related events take place within a reasonable time (for example weeks, months).

- If, in a reasonable time, hundreds of events favorable of accidents can occur, they will not lead to a catastrophic accident. But long time favors the hazard.

- A consequence of the large numbers law could be as follows: "A crash event can happen if the unfolding takes place over a long period and the number of events accumulates beyond the limit".

- The relation (3) that defines the law of large numbers can be interpreted differently: A very rare but not impossible event, with the probability of " $p$ ", can occur if a large enough number of attempts is made " $n$ " of which " $m$ " attempts are successful. The above interpretation and relationship allow us to determine in certain assumptions the number of attempts $n$ as a rare event to occur, and from here, until we expect the event to occur if a number of events $k$ occurs in a given time, one month, (deviations from compliance with security regulations).

$$
T_{m}=\frac{m}{k(p+\varepsilon)}
$$

The relationship allows the calculation of the duration in months $\left(T_{m}\right)$ after which the event is likely to reappear.

Let us consider a hypothetical case of producing an explosion in a location that seemed safe and determine the value $\mathrm{n}$ and hence the probable time to repeat the event. In the hypothesis $p=10^{-3}, \varepsilon=10^{-3}, m=1, k=15$ results $T_{m}>$ 33.3 months, $T_{m}=2.7$ years.

It follows from the above that the protection measures need to be further improved, which should be treated with all seriousness. Applying the probabilistic strategies act for increasing the duration of repetition or the complete elimination of the phenomenon through measures such as:

- Performing equipment with few defects and requiring a small number of interventions.

- Qualified staff with great expertise in interventions.

- Improving the SCADA scanning system by using the WSN sensor networks.

- Resetting the Probabilistic Events Field to eliminate the cumulative number of events that cause non-compliance with security conditions. 
- This last measure involves changing the monitoring and control strategy by using the Internet of Things (IoT) alternative. Some mining companies have also announced that they are in the advanced phase of implementing [10] [11] [12].

\section{Modeling/Simulation and WSN Implementation}

Below we will present an example of modeling/simulation and implementation of a WSN network in the case of underground coal extraction.

Figure 2 shows an example of coal extraction using mechanized support (a) and the process monitoring scheme (b) containing the SCADA and the WSN network. Such an approach limits the risk of accidents through the SCADA scanning system of the $\mathrm{S}$ sensors and provides a more secure location locally through the WSN network of the W sensors [13]. The modeling/simulation it was implemented on the CupCarbon platform as follows [14].

The real world of the mining site was designed based on the technological model shown in Figure 2 together with the SCADA S sensors.

Based on human experience, the methane from the free space 5 , the coal front 1 , the hopper 4 and the conveyor 6 were determined. In this way, a mobile element, simulating the evolution of methane, runs in this way. In this regard, the three WSN Wi $(i=1,2,3)$ sensors arranged in a star topology and transmitting to a final router connected to IoT were implemented. The model is shown in Figure 4 and the simulation results are shown in Figure 5 and Figure 6 for two

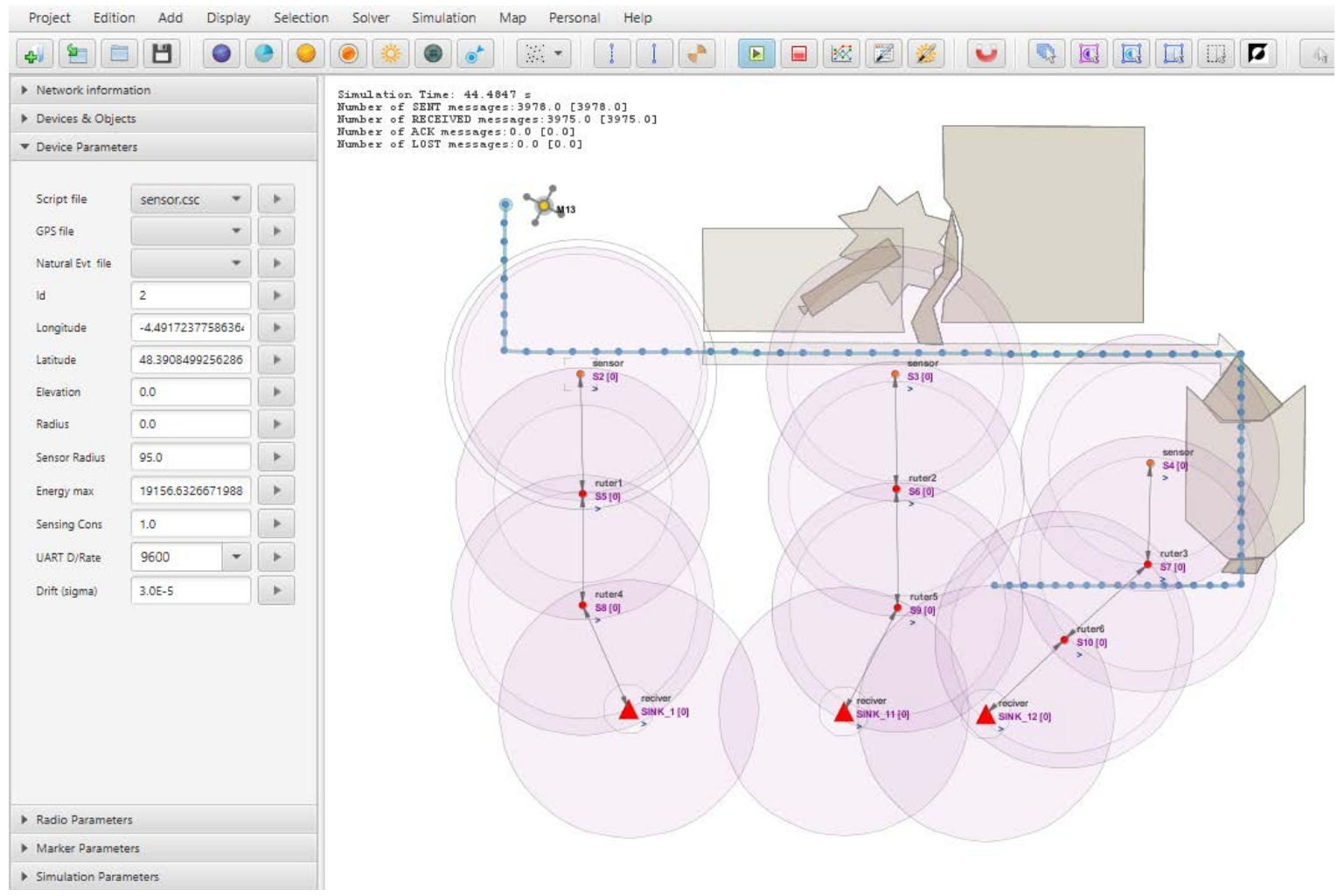

Figure 4. Model with WSN sensors in star connection. 


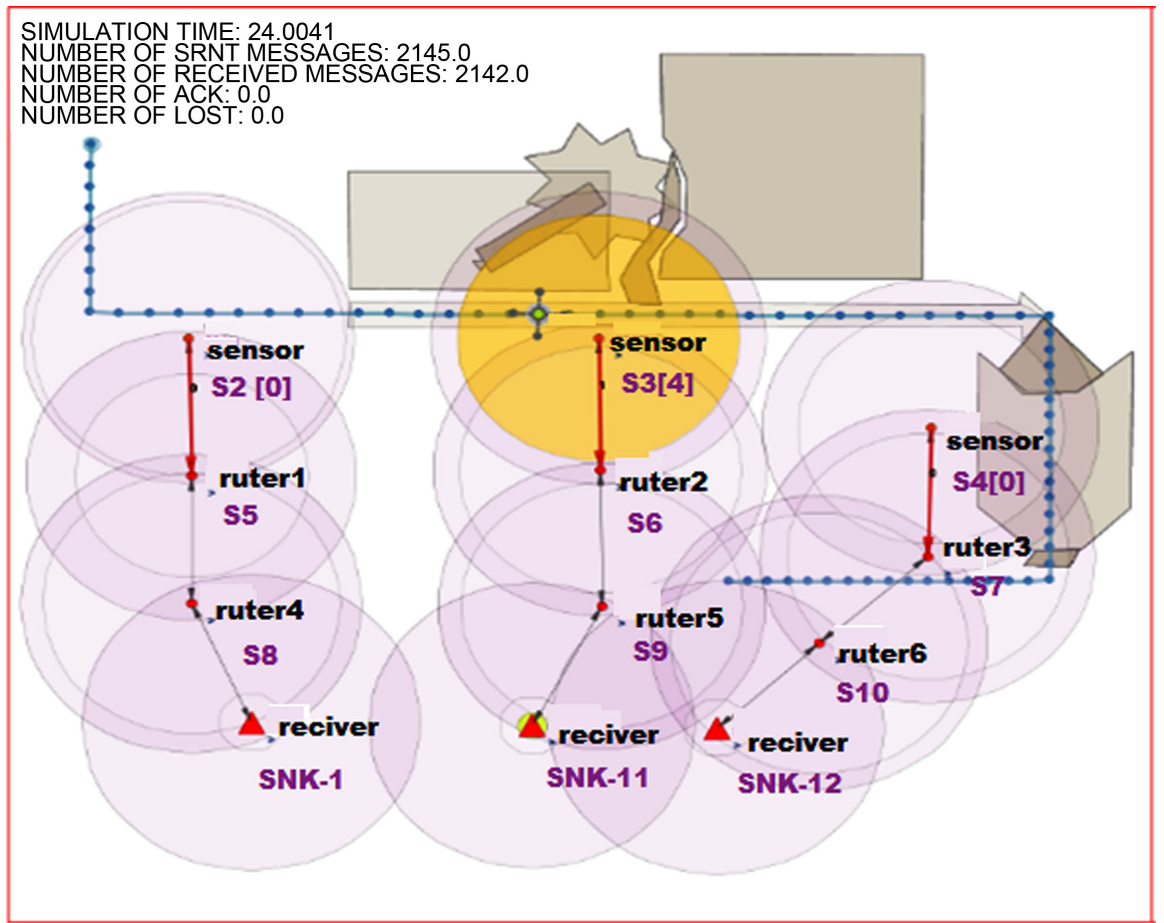

Figure 5. Simulation results for methane emission: (a) combine cutting.

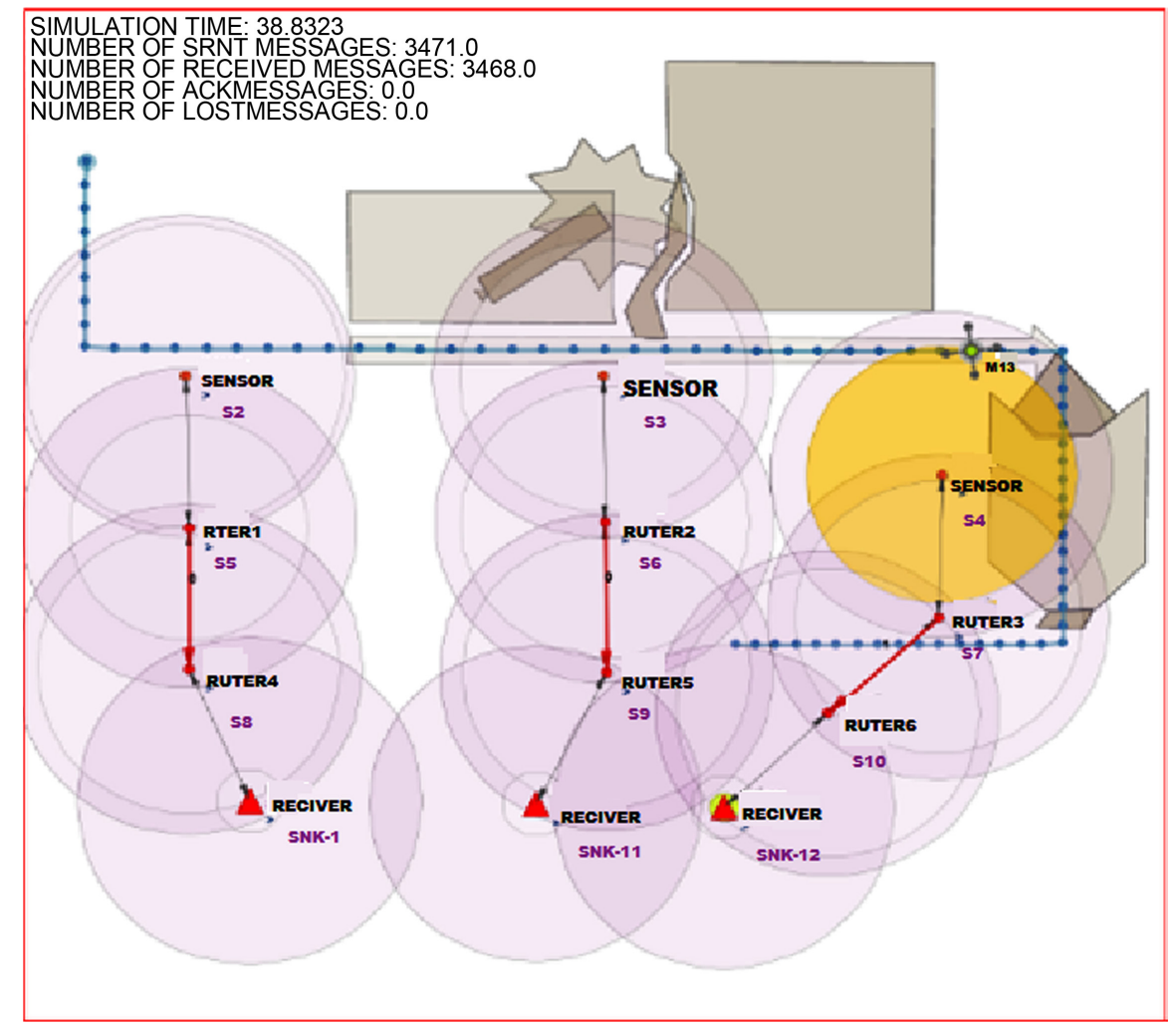

Figure 6. Simulation results for methane emission: (b) conveyer and silos.

situations: first when the intermediate sensor 2 detects the methane from the coal massive after cutting with the combine and the second when methane 
comes from bunker and the belt conveyor. The two sensors are programmed to receive data from the work environment, to cooperate with each other and to transmit them to the gateway's final receiver. The receiver is programmed to retrieve the data and upload it to the internet or IoT [15] [16].

Thus, process managers and coordinators get real-time information and decide what they need to do. Finally, the model for the simulation results is run and code is generated for implementation on the Arduino platform [17] [18] [19].

Figure 5 and Figure 6 show the simulation of monitoring and control of the potentially explosive location shown in Figure 2 using a WSN network with 3 -star sensors for methane detection at combine cutting and methane emission from silos [20] [21] [22]. The implementation was done using 3 methane sensors made with MQ-2 type camera and Arduino equipment.

To extract the information from the coal massive, to the main gallery, two routers were connected to each sensor, as presented in the simulation scheme.

The block diagram and deployment scheme are shown in Figure 7 and contain 3 sensors, 6 routers and 1 receiver, the latter being the gateway for the Internet or IoT connection [23].

\section{Conclusions}

- The explosion triangle consists of the existence in the atmosphere of the following conditions: 1) certain highly flammable substances, such as methane; 2) enough oxygen to keep the explosion; 3 ) sources of energy to initiate the explosions.
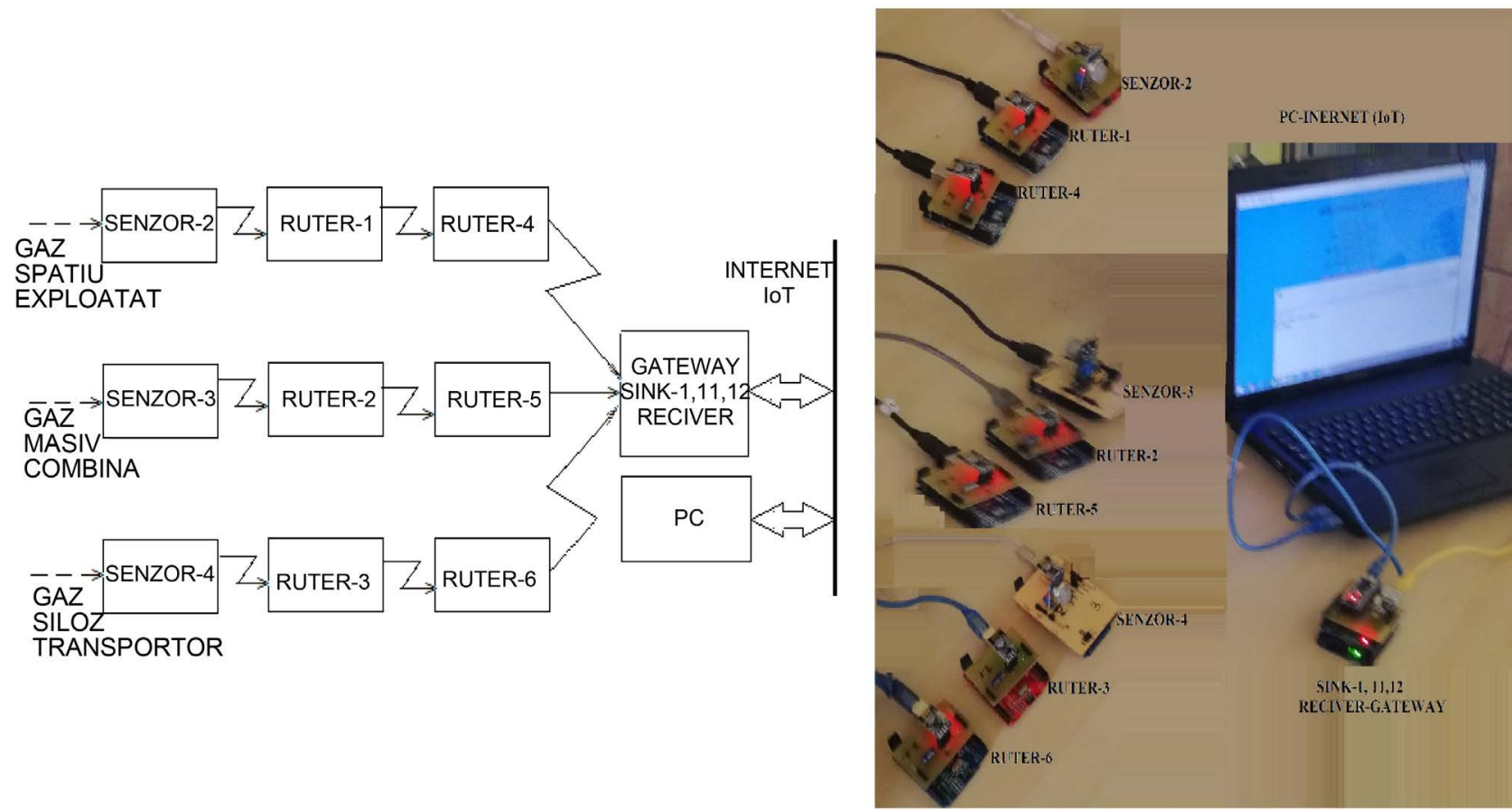

Figure 7. Control with WSN: (a) block diagram; (b) implementation scheme. 
- Potentially explosive atmospheres in the case of the first two conditions.

- Risk of explosion or hazardous area if all three conditions are present.

- A safe area where explosion cannot occur and cannot be transmitted outdoors to initiate another explosion.

- The risk of explosion can occur only in the following coincidence: there is methane in a hazardous percentage and there is a source of ignition.

- Deterministic strategy is a method to conserve the safe location through eliminating the risk of explosion. Severe methane removal measures are taken by continuous ventilation, maintaining a non hazardous inferior boundary. This limit is monitored by continuous scanning using a centralized SCADA system. Equally, the equipment is periodically inspected to have unaltered safety standards (no sources of ignition).

- Deterministic strategy has greatly reduced the explosion hazards but has not completely removed them so that unfortunately such incidents are still occurring from time to time.

- The "random phenomena", where rare events can occur, are favored by the complexity of some processes like from underground. The random phenomena cannot be analyzed through deterministic methods but through the "hazard laws". They are the limits of "deterministic strategy".

- A probabilistic strategy is a new method proposed in this paper based on Hazard laws like "Gauss's bell” and "The law of large numbers".

- The probabilistic strategy allows extending the safety of the personnel beyond the limits provided by the deterministic strategy.

- The theoretical results are validated by an application using simulation through CupCarbon platform, WSN sensors and Arduino equipments.

\section{Conflicts of Interest}

The authors declare no conflicts of interest regarding the publication of this paper.

\section{References}

[1] Pop, E., Ilcea, G. and Popa, I.A. (2018) Distance Control and Positive Security for Intrinsic Equipment Working in Explosive Potential Atmospheres. Engineering, 10, 75-84. https://doi.org/10.4236/eng.2018.103006

[2] Pop, E. (1983) Automatizari in indusreia miniera. EDP Publishing House, Bucharest.

[3] Amar, N.S., Ravi, R.K., Vijay, K. and Vipin, G. (2016) Safety of Underground Coal Mine Using Artificial Intelligence and Wireless Sensor Network. International Journal of Advanced Trends in Computer Science and Engineering, 5, 18940-18942.

[4] Markham, A. and Trigoni, N. (2012) Magneto-Inductive Networked Rescue System (MINERS), Taking Sensor Networks Underground. ACM/IEEE 11 th International Conference on Information Processing in Sensor Networks (IPSN), Beijing, 16-20 April 2012, 317-328. https://doi.org/10.1109/IPSN.2012.6920946

[5] Zhang, Y., Yang, W., Han, D. and Kim, Y. (2014) An Integrated Environment Monitoring System for Underground Coal Mines-Wireless Sensor Network Subsystem 
with Multi-Parameter Monitoring. Sensors, 14, 13149-13170. https://doi.org/10.3390/s140713149

[6] Bradly, A. and Automation, R. (2005) SCADA System Application Guide. Pub. AG-UM08C-EN-C, USA.

[7] https://cronicavj.ro/wp/?p=68908

[8] Centrala telegrizumetrică CTT 63/40 U. (2016) https://www.researchgate.net

[9] Căbulea, L., Luca-Tudorache, R., Pitea, A.G., Zbăganu, G., Rasa, I. and Breaz, N. (2016) Probabilități şi statistic. POSDRU/56/1.2/S/32768, Bucuresti.

[10] Norwegian-African Cooperative Project (2017) Wireless Network of Sensors Aimed at Preventing Explosions In Mines. Council for Scientific and Industrial Research, University of Pretoria, Pretoria. https://www.sintef.no/en

[11] Mine Site Technologies (MST) (2016) New Wireless Sensor Network Sets the Benchmark for IoT in Mining. https://www.mining.com

[12] Startup Licenses UA, Tech Launch Arizona (2018) Invented Mining Sensor Network. College of Engineering and Institute for Mineral Resources, University of Arizona, Tucson. https://uanews.arizona.edu

[13] Song, W. and Qiao, J. (2015) Research of Mine-shaft Wireless Sensor Network Based on Agent. International Conference on Education, Management, Commerce and Society (EMCS 2015), College of Information Engineering, Tayshaun Medical University, Taian, 29-31 January 2015, 563-569.

[14] CupCarbon ${ }^{\circledast}$ User Guide, Version U-One 3.8, France (2017) https://www.cupcarbon.com

[15] Friedewald, M. and Raabe, O. (2011) Ubiquitous Computing: An Overview of Technology Impacts, Telematics and Informatics. Elsevier, Vol. 28, 55-65.

[16] Ibrahiem, M.M., Emary, E. and Ramakrishnan, S. (2016) Wireless Sensor Networks: From Theory to Applications. CRC Press, Boca Raton.

[17] ZigBee \& RFID (2018) Interfețe pentru comunicații wireless. https://www.rfid-wiot-search

[18] Woo, A. (2018) MICA: The Commercialization of Microsensor Motes, Sensors Expo 2018.

[19] The Arduino Uno Is a Microcontroller Board Based on the ATmega328. https://datasheet.octopart.com

[20] Jeffree, T. (2011) IEEE 802.1 Standards Workshop, Singapore.

[21] Nandini, M., Sarmistha, N. and Sarbani, R. (2015) Building Wireless Sensor Networks: Theoretical and Practical Perspectives. CRC Press, Boca Raton.

[22] Raghavendra, C.S., Sivalingam, K.M. and Znati, T. (2004) Wireless Sensor Networks, Library of Congress. Springer, Berlin.

[23] International Electrotechnical Commission (2014) Internet of Things: Wireless Sensor Networks. Geneva. http://www.iec.ch 